\title{
Coulomb Blockade without Tunnel Junctions
}

\author{
Yuli V. Nazarov \\ Faculty of Applied Physics and DIMES, Delft University of Technology, Lorentzweg 1, 2628 CJ Delft, \\ The Netherlands
}

\begin{abstract}
We find that tunnel junctions are not needed to provide single electron effects in a metallic island. Eventually the tunnel junction may be replaced by an arbitrary scatterer. It is important that even a diffusive scatterer provides a sufficient isolation for single electron effects to persist. To formulate this in exact terms, we derive and analyze the effective action that describes an arbitrary scatterer. We also consider the fluctuations of the effective charging energy.
\end{abstract}

73.23.Hk, 73.23.Ps, 73.40.Gk

It is well-known that electric charge of an isolated piece of conducting material can only take discrete values corresponding to the integer number of electrons in there. This property persists if this isolated piece, the island, is connected to electron reservoir by means of a resistive tunnel junction It is the recognition of this mere fact that lead to outburst of the entire field of single electron phenomena. [i]

The single electron effects are best visible provided the conductivity of the tunnel junction is much smaller than the conductance quantum $G_{Q} \equiv e^{2} / 2 \pi \hbar$. The ground state energy as a function of induced charge $q$ is given by minimization of Coulomb energy, $E_{C}(n+q)^{2}$, with respect to discrete charge $n$. The result is periodic in $q$ with a period $e$. [1] The analysis of the reverse case, $G \gg G_{Q}$, requires advanced theoretical methods. [2 1 [ Despite the partial controversy in results, all authors agree that in this case the ground state energy retain the periodic $q$ dependence, that manifests the Coulomb blockade. The effective charging energy, $\tilde{E}_{C}$, that is, the $q$-dependent part of the ground state energy, is suppressed by a factor of $\exp \left(-G / 2 G_{Q}\right)$ in comparison with $E_{C}$.

Still the analysis has been restricted to tunnel junctions. Next step has been made in [5:,6] where Coulomb blockade has been studied in the situation where the isolation is provided by a quantum point contact with almost perfect transparency. It has been shown that the charge quantization survives. Albeit the charging energy is strongly suppressed vanishing to zero at perfect transmission.

In this paper, we construct a general theory of Coulomb blockade that can embrace tunnel junctions, quantum point contacts, diffusive conductors and eventually any type of scattering.

The results are as follows. Charging energy vanishes only for perfect point contacts. For very wide class of conductors that have conductivity $G \gg G_{Q}$, the charging energy is exponentially suppressed, $\ln \left(\tilde{E}_{C} / E_{C}\right) \propto-\alpha G / G_{Q}, \alpha$ being dimensionless coefficient depending on the type of the conductor. For disordered conductors, for instance, diffusive ones, the charging energy strongly fluctuates. This happens even if the fluctuations of the conductance are small.

The most equivalent mathematical framework to describe the charging effects in full has been proposed by Schön and Zaikin. [7] They present the partition function of the system in the form of the path integral over the field $\varphi(\tau)(\beta=\hbar / T)$,

$$
Z=\int \prod_{\tau} d \varphi(\tau) \exp \left(-\mathcal{L}_{s c}[\varphi(\tau)]+\int_{0}^{\beta} d \tau\left(-\frac{\dot{\varphi}(\tau)^{2}}{2 E_{C}}-i \frac{q \dot{\varphi}(\tau)}{e}\right)\right.
$$

The form of the last term presumes that the partition function can be presented as a sum over topological sectors that are labelled by an integer $W=\int_{0}^{\beta} d \tau \dot{\varphi}(\tau)$ winding number of $\varphi(\tau)$,

$$
Z=\sum_{W} Z_{W} \exp (-i 2 \pi W q / e)
$$

Here $Z_{W}$ does not depend on $q$.

The action $\mathcal{L}_{s c}$ describes tunnel junction. It can be evaluated with using Tunneling Hamiltonian method that gives

$$
-\mathcal{L}_{s c}=\frac{G_{T}}{2 \beta^{2} G_{Q}} \int_{0}^{\beta} d \tau \int_{0}^{\beta} d \tau^{\prime} \frac{\sin ^{2}\left(\left(\varphi(\tau)-\varphi\left(\tau^{\prime}\right)\right) / 2\right)}{\sin ^{2}\left(\pi\left(\tau-\tau^{\prime}\right) / \beta\right)} .
$$

This form shows that the tunnel junction is quite different form a linear resistor which is described by a form

$$
-\mathcal{L}_{s c}=\frac{G}{8 \beta^{2} G_{Q}} \int_{0}^{\beta} d \tau \int_{0}^{\beta} d \tau^{\prime} \frac{\left(\varphi(\tau)-\varphi\left(\tau^{\prime}\right)\right)^{2}}{\sin ^{2}\left(\pi\left(\tau-\tau^{\prime}\right) / \beta\right)}
$$


which is bilinear in $\varphi$. It has been frequently assumed that a coherent diffusive conductor can be described by (4) and consequently exhibits no charging effects. We show below that it is not so. However, the relation (4) holds for an arbitrary conductor in the limit of small $\varphi$.

We sketch the derivation of $\mathcal{L}_{s c}$ for an arbitrary conductor. Our basic assumptions are: (i) no inelastic scattering occurs in the conductor, (ii) the conductor is sufficiently short, $E_{C} \tau_{\text {trav }} / \hbar \ll 1, \tau_{\text {trav }}$ being typical traversal time throught the conductor, (iii) the island is sufficiently large, so that $E_{C}$ greatly exceeds the average level spacing in the island. Two first assumptions allow us to characterize the conductor by an elastic scattering matrix disregarding retardation effects. The third assumption allows us to disregard coherence between the electrons transmitted to and coming from the island, so that it can be regarded as an electron reservoir.

These assumptions correspond to a fermionic action of the following form:

$$
\begin{array}{r}
-\mathcal{L}=\int_{0}^{\beta} d \tau\left(\sum_{n=1}^{N} \int_{-\infty}^{0} d x \chi_{n}^{\dagger}(x, \tau)\left[\partial_{\tau}+i v_{n} \partial_{x}\right] \chi_{n}(x, \tau)+\psi_{n}^{\dagger}(x, \tau)\left[\partial_{\tau}-i v_{n} \partial_{x}\right] \psi_{n}(x, \tau)\right. \\
+\sum_{m=1}^{M} \int_{0}^{\infty} d x \chi_{n}^{\dagger}(x, \tau)\left[\partial_{\tau}-i v_{n} \partial_{x}\right] \chi_{n}(x, \tau)+\psi_{n}^{\dagger}(x, \tau)\left[\partial_{\tau}+i v_{n} \partial_{x}\right] \psi_{n}(x, \tau) \\
\left.+E_{C}(Q(\tau)-q / e)^{2}\right)
\end{array}
$$

Here the island is on the right $(x>0), n$ and $m$ label transport channels in the island and the reservoir respectively. $\chi$ stands for fermion fields coming to the scatterer and $\psi$ stands for outgoing modes. The scatterer is completely characterized by the scattering matrix $\hat{S}_{k l}$ that set a boundary condition for $\psi$ and $\chi$

$$
\psi_{k}(0)=\sum_{l} S_{k l} \chi_{l}(0)
$$

Here $k, l$ label modes on both sides of the scatterer.

The charge in the island is given by

$$
Q(\tau)=\sum_{m=1}^{M} \int_{0}^{\infty} d x\left(\chi_{m}^{\dagger}(x, \tau) \chi_{m}(x, \tau)+\psi_{m}^{\dagger}(x, \tau) \psi_{m}(x, \tau)\right)
$$

To proceed, we perform a Hubbard-Stratanovitch transform on interaction term introducing a new variable $\varphi(\tau)$,

$$
\left.E_{C}(Q(\tau)-q / e)^{2}\right) \rightarrow-\frac{\phi \dot{(\tau)^{2}}}{2 E_{C}}+i Q(\tau) \dot{\phi(\tau)}-i q / e \dot{\phi}(\tau)
$$

The resulting action is quadratic in fermions so that they can be integrated out and the action can be represented as a functional of $\varphi(\tau)$. It is constructive to do this calculating Green functions of the fermions in the presence of field $\phi$ and scattering potential. Special attention shall be given to the fact that in one dimension the Green function $G\left(x, \tau, x^{\prime}, \tau^{\prime} ;[\varphi(\tau)]\right)$ is not continuous at coinciding arguments. So that the problem shall be regularized by letting scattering to occur in small but finite region of space. We present the action as a series in $\Delta G=G_{0}\left(x, \tau, x^{\prime}, \tau^{\prime} ;[\varphi(\tau)=\right.$ $0])-G_{0}\left(x, \tau, x^{\prime}, \tau^{\prime} ;[\varphi(\tau)]\right)$ where $G_{0}$ refers to Green functions of disconnected island and reservoir, since $\Delta G$ is continuous. Then the action can be reduced to the trace of logarithm of an operator,

$$
-\mathcal{L}_{s c}=\operatorname{Tr}_{n, \tau} \ln \left(1-(1-\hat{r}) \hat{\theta}^{+} \exp (-i \varphi) \hat{\theta}^{-} \exp (i \varphi)-\left(1-\hat{r}^{+}\right) \hat{\theta}^{-} \exp (-i \varphi) \hat{\theta}^{+} \exp (i \varphi)\right)
$$

The operator is defined in a Gilbert space which is the direct product of the Gilbert space of the transport channels in the island and the Gilbert space of fermion functions in imaginary time. The operator $\hat{r}$ is the reflection matrix in the island. The operators $\hat{\theta}^{ \pm}$are projection operators on positive (negative) Matsubara frequencies, $\hat{\theta}^{ \pm} \equiv \theta\left( \pm \epsilon_{n}\right)$. Although $\hat{\theta}^{ \pm}$obviously commute with $\hat{r}, \hat{r}^{+}$, they do not commute with multiplication by $\exp ( \pm i \varphi(\tau))$. This generates a complicated operator algebra that makes a complicated problem even the evaluation of $\mathcal{L}_{s c}$.

The expression (9) can be explicitly evaluated in two limits: $\hat{r} \rightarrow 1$ and $\phi \rightarrow 1$. There, we successfully reproduce Eq. 3 for tunnel junctions and Eq. 4 for an arbitrary scatterer in linear regime.

Fortunately, a very important part of analysis of the action (9) can be done exactly. We are able to find the minima of (9) in each topological sector and thus give a quantitative estimate of effective Coulomb energy in the limit $G \gg G_{Q}$. We consider the configurations of $\varphi(\tau)$ of the following form: 


$$
\exp (i \varphi)=\prod_{i=1}^{N} \frac{u-z_{i}}{1-u z_{i}^{*}} .
$$

Here $u \equiv \exp (i 2 \pi \tau / \beta), z_{i}$ are complex parameters. $z_{i}$ can be viewed as coordinates of $N$ (anti)solitons in the plane of complex $u$ (see Fig.2). If $\left|z_{i}\right| \rightarrow 1$, these configurations correspond to sets of Korshunov's solitons. [B] We are interested in configurations where all solitons are of the same sign. In this case either $\left|z_{i}\right|<1$ for all $i$ or $\left|z_{i}\right|>1$ so that $\exp (i \varphi)$ is an analytical function of $u$ either within or beyond the unitary circle. The winding number $W= \pm N$. Using the methods of analytical function theory we show that these configurations indeed minimize the action in the corresponding topological sector. The minimum does not depend on $z_{i}$ and equals

$$
-\mathcal{L}_{W}=\frac{1}{2} \ln \operatorname{det}\left(\hat{r} \hat{r}^{+}\right)|W|+\frac{1}{2} \ln \operatorname{det}\left(\hat{r} / \hat{r}^{+}\right) W .
$$

The second term is imaginary and can be viewed as a trivial shift of induced charge $q: q \rightarrow q+i \ln \operatorname{det}(\hat{r} / \hat{r}+)$. The first term is of importance since it describes the suppression of statistical weight of topogical sectors with $W \neq 0$ in comparison with the trivial sector. It has been shown in [2.3] that the suppression of these statistical weights leads to suppression of effective charging energy. This allows us to write down a simple formula for effective charging energy

$$
\tilde{E}_{C} \propto E_{C} \prod_{n} R_{n}^{1 / 2}
$$

where $R_{n}$ are eigenvalues of the reflection matrix $\hat{r} \hat{r}^{+}$. This formula is valid provided the suppression is big. Similar relation has been obtained by Flensberg [6 in a much more restrictive framework. In the limit of almost perfect transmission, $R \rightarrow 0$, we reproduce the results of Matveev [5].

Recent theoretical advantages allow to characterize $R_{n}$ of a scatterer/conductor of virtually any type (see [9] for review). This makes the relation (12) easy to use for concrete examples. From now on, we will concentrate on diffusive conductor in the limit $G \gg G_{Q}$. It is a disordered conductor, so that it is characterized by distribution of $R_{n}$, or transmissions $T_{n}=1-R_{n}$. It has been shown in 10 that the transmission distribution of a diffusive conductor depends only on its conductance, $\rho(T)=G /\left(2 G_{Q} T \sqrt{1-T}\right)$. We average logarithm of (12) with this distribution to obtain

$$
\tilde{E}_{C} / E_{C} \propto \exp \left(-\frac{\pi^{2} G}{8 G_{Q}}\right) .
$$

This is the main result of this work. The diffusive scatterer of the same resistance as a tunnel junction suppresses Coulomb blockade much more efficiently. To give some numbers, let us choose $1 / G=4 \mathrm{kOhm}$. In this case, suppression factor is about 25 for a tunnel junction and almost 3000 for a diffusive conductor.

Below we consider the fluctuations of $\tilde{E}_{C}$ and the effect of weak localization. To make a qualitative estimation, we note that the fluctuation of $G$ is of the order of $G_{Q}$. The weak localization correction is of the same scale. Therefore, the fluctuations of an exponential like (13) must be of the order of its average value. The same should hold for the weak localization effect. It is remarkable that quantitative consideration gives even bigger values.

This quantitative treatment can be performed along the lines of 11] and [12. There are formulas that can be directly applied to the quantity of interest $\ln \left(\tilde{E}_{C} / E_{C}\right)=\sum_{n} \ln R_{n}$. It appears that both the fluctuation and the localization effect are dominated by a contribution of the universal cooperon-diffusion mode, the one which provides Wigner-Dyson statistics of closely spaced transmission eigenvalues. [11] The contribution of this mode logarithmically diverges at very small $R$ and shall be cut off at $R \simeq G_{Q} / G$, average value of transmission spacing. For pure statistical ensembles, the fluctuation is given by

$$
<<\ln ^{2}\left(\tilde{E}_{C} / E_{C}\right)>>=\frac{N_{d c}}{4} \ln \left(G / G_{Q}\right)
$$

where $N_{d c}$ is the number of massless cooperon and diffusion modes. It ranges from 1 to 8 . The weak localization correction is

$$
<\ln \left(\tilde{E}_{C} / E_{C}\right)>_{w l}=-\frac{N_{w l}}{4} \ln \left(G / G_{Q}\right)
$$

where $N_{w l}$ is 2, 0, -1 for simplectic, unitary and orthogonal ensemble respectively.

Experimentally, the fluctuation and weak localization effect are identified by their magnetic field dependence. Following [11] we introduce dimensionless parameters $\eta_{H}, \eta_{S O}$ to characterize magnetic field and spin-orbit interaction. 
We disregard influence of magnetic field on spin. Correlator of two $\tilde{E}_{C}$ taken at different values of magnetic field reads

$$
<<\ln \left(\tilde{E}_{C}\left(\eta_{H}\right) / E_{C}\right) \ln \left(\tilde{E}_{C}\left(\eta_{H}^{\prime}\right) / E_{C}\right)>>=-\frac{1}{4} \ln \left(\left|\eta_{H}^{2}-\eta_{H}^{\prime 2}\right|^{4}\left(\left(\eta_{H}+\eta_{H}^{\prime}\right)^{2}+\eta_{S O}^{2}\right)^{3}\left(\left(\eta_{H}-\eta_{H}^{\prime}\right)^{2}+\eta_{S O}^{2}\right)^{3}\right) .
$$

This equation is valid provided $G / G_{Q} \ll\left|\eta_{H}-\eta_{H}^{\prime}\right|, \eta_{H}, \eta_{H}^{\prime}, \eta_{S O} \ll 1$. It shows that the actual value of $\tilde{E}_{C}$ can be changed by an order of magnitude by fairly small change of magnetic field $\delta \eta_{H} \simeq G_{Q} / G$. At a bigger $\delta \eta_{H}$ effective charging energy exhibits power-law correlations.

Magnetic field dependence of the weak localization correction is given by

$$
<\ln \left(\tilde{E}_{C} / E_{C}\right)>_{w l}=\frac{1}{4}\left(3 \ln \left(\eta_{H}^{2}+\eta_{S O}^{2}\right)-\ln \left(\eta_{H}^{2}\right)\right) .
$$

To conclude, we have shown that the isolation required for discrete charge effects can be provided by any constriction which is not ideally ballistic. We have discussed suppression of the effective charging energy by a diffusive scatterer and found gigantic fluctuations of this quantity.

I am indebted to G. W. E. Bauer, P. W. Brouwer, S. E. Korshunov, Y. Gefen and many others for very instructive discussions of the results. This work is a part of the research programme of the "Stichting voor Fundamenteel Onderzoek der Materie" (FOM) and I acknowledge the financial support from the "Nederlandse Organisatie voor Wetenschappelijk Onderzoek" (NWO).

[1] Single Charge Tunneling, eds. H. Grabert and M. H. Devoret, NATO ASI Series B 294 (Plenum, New York, 1992), the whole book.

[2] S. V. Panyukov and A. D. Zaikin, Phys. Rev. Lett.67 3168 (1991).

[3] X. Wang and H. Grabert, Phys. Rev. B 53, 12621 (1996).

[4] W. Hofstetter and W. Zwerger, Phys. Rev. Lett. 78, 3737 (1997).

[5] K. A. Matveev, Phys. Rev. B 51, 1743 (1995).

[6] K. Flensberg, Phys. Rev. B 48, 11156 (1993).

[7] G. Schön and A. D. Zaikin, Phys. Rep. 198, 237 (1990).

[8] S. E. Korshunov, Pisma Zh. Eksp. Teor. Fiz 45, 342 (1987) [JETP Lett. 45, 434 (1987)].

[9] C. W. J. Beenakker, Rev.Mod.Phys. 69, 731 (1997)

[10] Yu. V. Nazarov, Phys. Rev. Lett., 73, 134 (1994).

[11] Yu. V. Nazarov, Phys. Rev. Lett. 76, 2129 (1996).

[12] Yu. V. Nazarov, Phys. Rev. B 52, 4720 (1995). 